\title{
A MULTIPLICATIVE ANALOGUE OF THE REYNOLDS OPERATOR AND CONSTRUCTION OF INVARIANTS
}

\author{
STEVEN DECKELMAN, JENNIFER GRAETZ AND TYLER RUSSELL
}

\begin{abstract}
We explore a multiplicative analogue of the Reynolds operator that first arose in CR geometry. Comparisons with the classical Reynolds operator are made in connection with the problem of computing algebra generators in invariant rings over $\mathbb{C}$.
\end{abstract}

1. Introduction. Let $\Gamma$ be a finite group of unitary matrices on $\mathbb{C}^{n}$. For $z \in \mathbb{C}^{n}$, the Hermitian symmetric polynomial

$$
\Phi(z, \bar{z})=1-\prod_{\gamma \in \Gamma}(1-\langle\gamma z, z\rangle)
$$

was introduced by D'Angelo in [3] to construct group-invariant CR mappings from the sphere to hyperquadrics in CR geometry. CR mappings are mappings between CR manifolds, manifolds that contain some of the structure of complex manifolds. A typical example arises from the restriction of holomorphic mappings to a real submanifold of Euclidean space. The term CR stands for "Cauchy-Riemann" or, alternatively, "complex-real," see $[\mathbf{1}, \mathbf{2}, \mathbf{3}]$. In [5], it was shown that the ring of all $\Gamma$ invariant polynomials is generated as an algebra by the components of $F \bigoplus G$ where $\Phi(z, \bar{z})=\|F(z)\|^{2}-\|G(z)\|^{2}$. Here $F \bigoplus G$ is a holomorphic polynomial map $\left(f_{1}, f_{2}, \ldots, f_{N_{+}}, g_{1}, g_{2}, \ldots, g_{N_{-}}\right)$formed from two maps $F=\left(f_{1}, f_{2}, \ldots, f_{N_{+}}\right)$and $G=\left(g_{1}, g_{2}, \ldots, g_{N_{-}}\right)$determined by $\Phi$. The process of writing $\Phi(z, \bar{z})$ as a difference of two squared norms of polynomial maps with linearly independent components is called holomorphic decomposition and amounts to diagonalizing an Hermitian matrix. See [3, 4] for more on holomorphic decomposition. In [5], it was asserted without proof that the result about genera-

2010 AMS Mathematics subject classification. Primary 13A50, 20C30, 20C40, 32 V99.

This research was supported by NSF Grant No. 1062403.

Received by the editors on July 24, 2012, and in revised form on February 23, 2013. 
tors should generalize to arbitrary non-unitary finite groups. Our main result is to give a proof of this and describe the algorithm it suggests for the computation of algebra generators in invariant rings. We do this by essentially reducing the general case to the unitary case. This result takes an especially simple form in the case of finite abelian groups.

2. Background and definitions. Let $G L(n, \mathbb{C})$ denote the group of $n \times n$ nonsingular matrices. By a representation of a finite group $G$ we mean a homomorphism

$$
\rho: G \rightarrow G L(n, \mathbb{C}) .
$$

In this paper we will always work with the image of such a representation, a finite matrix group we will usually denote by $\Gamma=\rho(G)$. Such a representation or matrix group induces an invariant ring that we denote by

$$
\mathbb{C}[z]^{\Gamma}=\{f \in \mathbb{C}[z] \mid f(\gamma z)=f(z), \quad \text { for all } \gamma \in \Gamma\} .
$$

Note that $\gamma$ is a matrix, $z=\left(z_{1}, z_{2}, \ldots, z_{n}\right)$ and $\mathbb{C}[z]$ is a polynomial ring. [9] is a good reference on these ideas. These rings are actually algebras and are finitely generated by the Hilbert finiteness theorem. By a set of generators for $\mathbb{C}[z]^{\Gamma}$, we mean a finite set of polynomials

$$
f_{1}, f_{2}, \ldots, f_{k} \in \mathbb{C}[z]^{\Gamma}
$$

such that

$$
\mathbb{C}[z]^{\Gamma}=\mathbb{C}\left[f_{1}, f_{2}, \ldots, f_{k}\right]
$$

In other words, for each $g \in \mathbb{C}[z]^{\Gamma}$ there exists an integer $k$ and a complex polynomial $p$ in $k$ variables such that

$$
g(z)=p\left(f_{1}(z), f_{2}(z), \ldots, f_{k}(z)\right)
$$

In the older literature, such sets of algebra generators (2.1) were called complete fundamental systems of invariants or an integrity basis. We emphasize that our definition does not require (2.1) to be minimal. Neither do the polynomials in (2.1) need to be algebraically independent. The polynomials (2.1) simply generate the algebra $\mathbb{C}[z]^{\Gamma}$ in an analogous manner to the way that a (potentially non-minimal) spanning set of vectors might span a vector space. It is well known in invariant 
theory, however, that any such set of generators will contain a minimal generating set. See [9] for more details.

One of the classical problems is, given an invariant ring $\mathbb{C}[z]^{\Gamma}$, how to find or compute a set of generators such as (2.1). Historical studies of this problem centered on rings over fields of characteristic zero (e.g., $\mathbb{C}$ ). A good deal of modern research studies the modular case. In this paper all of our invariant rings are over the complex field $\mathbb{C}$. Our results do not apply to the modular case.

The Reynolds operator (sometimes called the averaging operator) is defined as

$$
R: \mathbb{C}[z] \longrightarrow \mathbb{C}[z], \quad f(z) \longmapsto \frac{1}{|\Gamma|} \sum_{\gamma \in \Gamma} f(\gamma z) .
$$

By a result of Emmy Noether, it is possible to find a generating set of polynomials for $\mathbb{C}[z]^{\Gamma}$ by applying the Reynolds operator to the set of all monomials $f \in \mathbb{C}[z]$ with $\operatorname{deg}(f) \leq|\Gamma|$. However, the set of these polynomials need not be a minimal set of invariants.

\section{Main result.}

Theorem 3.1. Let $\Gamma$ be a finite subgroup of $G L(n, \mathbb{C})$. Then there exists a $T \in G L(n, \mathbb{C})$ such that the polynomial defined by

$$
\Phi(z, \bar{z})=1-\prod_{\gamma \in \Gamma}(1-\langle T \gamma z, T z\rangle)
$$

has the property that the components of the map $F \oplus G$ arising from the holomorphic decomposition of $\Phi$ into $\|F\|^{2}-\|G\|^{2}$ with linearly independent components generate the invariant ring $\mathbb{C}[z]^{\Gamma}$.

Proof. For $\Gamma$ unitary, this result was proved in [5] with $T=I d$. In case $\Gamma$ is nonunitary, there exists a $T \in G L(n, \mathbb{C})$ so that

$$
\Gamma^{\prime}=T \Gamma T^{-1}
$$

is unitary. We recall the proof of this basic fact from linear algebra. See [10] for more details. Define

$$
B(z, w)=\frac{1}{|\Gamma|} \sum_{\gamma \in \Gamma}\langle\gamma z, \gamma w\rangle .
$$


Then $B(z, w)$ will be a $\Gamma$ invariant inner product in the sense

$$
B(\gamma z, \gamma w)=B(z, w)
$$

for all $\gamma \in \Gamma$. Assume $\left\{e_{1}, e_{2}, \ldots, e_{n}\right\}$ is the standard orthonormal basis for $\mathbb{C}^{n}$ equipped with $\langle\cdot, \cdot\rangle$. Let $\left\{f_{1}, f_{2}, \ldots, f_{n}\right\}$ be an orthonormal basis for $\mathbb{C}^{n}$ equipped with $B(z, w)$. Define the linear map $T: \mathbb{C}^{n} \rightarrow \mathbb{C}^{n}$ by $T\left(e_{i}\right)=f_{i}$, and let $P$ be its transition matrix, i.e.,

$$
P[v]_{f}=[v]_{e}
$$

where $[v]_{f},[v]_{e}$ are coordinates of $v \in \mathbb{C}^{n}$ in the two bases. Then

$$
B(z, w)=\left\langle[z]_{f},[w]_{f}\right\rangle
$$

with $[z]_{f}=P^{-1}[z]_{e}=P^{-1} z$ and $[w]_{f}=P^{-1} w$. In particular,

$$
B(z, w)=\left\langle P^{-1} z, P^{-1} w\right\rangle
$$

so we take $T=P^{-1}$. That $P^{-1} \gamma P$ is unitary follows from

$$
\left\langle P^{-1} \gamma P z, P^{-1} \gamma P w\right\rangle=\langle z, w\rangle
$$

Thus, the holomorphic decomposition into linearly independent components of

$$
1-\prod_{\gamma}\left(1-\left\langle T \gamma T^{-1} z, z\right\rangle\right)=\|F\|^{2}-\|G\|^{2}
$$

yields components of $F \bigoplus G$ as a set of algebra generators for $\mathbb{C}[z]^{\Gamma^{\prime}}$.

Since $\Gamma$ and $\Gamma^{\prime}$ are equivalent representations, the invariant rings $\mathbb{C}[z]^{\Gamma}$ and $\mathbb{C}[z]^{\Gamma^{\prime}}$ will be isomorphic with canonical isomorphism

$$
\tau: \mathbb{C}[z]^{\Gamma} \rightarrow \mathbb{C}[z]^{\Gamma^{\prime}}
$$

defined by $f \mapsto f \circ T^{-1}$ (i.e., $f \circ P^{-1}$ above). See [9] for more details. Of course, $\tau^{-1}(f)=f \circ T$. So

$$
(F \circ T) \bigoplus(G \circ T)
$$


will contain algebra generators for $\mathbb{C}[z]^{\Gamma}$. Equation (3.1) then follows by making a change of variables in equation $(3.2), z \mapsto T z$ :

$$
\begin{aligned}
\|(F \circ T)(z)\|^{2}-\|(G \circ T)(z)\|^{2} & =1-\prod_{\gamma}(1-\langle T \gamma z, T z\rangle) \\
& =\Phi(z, \bar{z}) .
\end{aligned}
$$

Theorem (3.1) now yields an algorithm for computing a set of generators for $\mathbb{C}[z]^{\Gamma}$. This follows from the properties of holomorphic decomposition and is described in greater detail in [5].

By a monomial in $\mathbb{C}[z]$, we mean a polynomial of the form

$$
z^{\alpha}=z_{1}^{\alpha_{1}} \ldots z_{n}^{\alpha_{n}}
$$

where $\alpha=\left(\alpha_{1}, \ldots, \alpha_{n}\right)$ and $|\alpha|=\alpha_{1}+\alpha_{2}+\cdots+\alpha_{n}$ is the usual multiindex or exponent sequence notation as in [9]. We say $z^{\alpha}$ appears in $\sum c_{\alpha \beta} z^{\alpha} \bar{z}^{\beta}$ if $c_{\alpha \beta} \neq 0$ for some $\alpha$.

Algorithm 3.2. 1. Expand (3.1) into the form $\sum c_{\alpha \beta} z^{\alpha} \bar{z}^{\beta}$.

2. Form the vector of appearing monomials $X$.

3. Compute the orthonormal eigenvectors $v_{i}$ of the Hermitian matrix $\left(c_{\alpha \beta}\right)$. (Unitarily diagonalize $\left(c_{\alpha \beta}\right)$.)

4. The set, $\left\langle X, v_{i}\right\rangle$, for all eigenvectors $v_{i}$ whose corresponding eigenvalues are nonzero give the algebra generators.

As an example of Algorithm 1, consider the following representation of the dihedral group of order 6 . This group is generated by

$$
\left(\begin{array}{cc}
\omega & 0 \\
0 & \omega^{-1}
\end{array}\right), \quad\left(\begin{array}{ll}
0 & 1 \\
1 & 0
\end{array}\right)
$$

where $\omega$ is a primitive cube root of unity. Since the group is unitary we may take $T$ to be the identity. In [7], Dusty Grundmeier used 
Mathematica to compute

$$
\begin{aligned}
\Phi(z, \bar{z})= & 1-\prod_{\gamma \in \Gamma}(1-\langle\gamma z, z\rangle) \\
= & z_{1}^{3}{\overline{z_{1}}}^{3}+z_{2}^{3}{\overline{z_{1}}}^{3}-z_{1}^{3} z_{2}^{3}{\overline{z_{1}}}^{6} \\
& +6 z_{1} z_{2}{\overline{z_{1} z_{2}}}^{4} 3 z_{1}^{4} z_{2}{\overline{z_{1}}}^{4}{\overline{z_{2}}}^{2}-3 z_{1} z_{2}^{4}{\overline{z_{1}}}^{4} \overline{z_{2}} \\
& -9 z_{1}^{2} z_{2}^{2}{\overline{z_{1}}}^{2}{\overline{z_{2}}}^{2}+z_{1}^{3}{\overline{z_{2}}}^{3}+z_{2}^{3}{\overline{z_{2}}}^{3} \\
& -z_{1}^{6}{\overline{z_{1}}}^{3}{\overline{z_{2}}}^{3}-z_{2}^{6}{\overline{z_{1}}}^{3}{\overline{z_{2}}}^{3} \\
& -3 z_{1}^{4} z_{2}{\overline{z_{1} z_{2}}}^{4}-3 z_{1} z_{2}^{4}{\overline{z_{1} z_{2}}}^{4}-z_{1}^{3} z_{2}^{3}{\overline{z_{2}}}^{6}
\end{aligned}
$$

The appearing monomials are $z_{1}^{3}, z_{2}^{3}, z_{1}^{3} z_{2}^{3}, z_{1} z_{2}, z_{1}^{4} z_{2}, z_{1} z_{2}^{4}, z_{1}^{2} z_{2}^{2}$, $z_{1}^{6}, z_{2}^{6}$. Ordering these lexicographically, we form the vector of appearing monomials

$$
X=\left(z_{2}^{3}, z_{2}^{6}, z_{1} z_{2}, z_{1} z_{2}^{4}, z_{1}^{2} z_{2}^{2}, z_{1}^{3}, z_{1}^{3} z_{2}^{3}, z_{1}^{4} z_{2}, z_{1}^{6}\right)
$$

and the $9 \times 9$ Hermitian matrix

$$
\left(\begin{array}{ccccccccc}
1 & 0 & 0 & 0 & 0 & 1 & 0 & 0 & 0 \\
0 & 0 & 0 & 0 & 0 & 0 & -1 & 0 & 0 \\
0 & 0 & 6 & 0 & 0 & 0 & 0 & 0 & 0 \\
0 & 0 & 0 & -3 & 0 & 0 & 0 & -3 & 0 \\
0 & 0 & 0 & 0 & -9 & 0 & 0 & 0 & 0 \\
1 & 0 & 0 & 0 & 0 & 1 & 0 & 0 & 0 \\
0 & -1 & 0 & 0 & 0 & 0 & 0 & 0 & -1 \\
0 & 0 & 0 & -3 & 0 & 0 & 0 & -3 & 0 \\
0 & 0 & 0 & 0 & 0 & 0 & -1 & 0 & 0
\end{array}\right)
$$

The six eigenvectors of this matrix corresponding to nonzero eigenvalues are $(0,0,0,1,0,0,0,0,1,0),(1,0,0,0,0,1,0,0,0),(0,1,0,0,0,0, \sqrt{2}$, $0,1),(0,1,0,0,0,0,-\sqrt{2}, 0,1),(0,0,0,0,1,0,0,0,0),(0,0,1,0,0,0,0,0$, $0)$.

Taking the inner product of (3.4) with these vectors give the invariants: $z_{1} z_{2}^{4}+z_{1}^{4} z_{2}, z_{2}^{3}+z_{1}^{3}, z_{2}^{6}+\sqrt{2} z_{1}^{3} z_{2}^{3}+z_{1}^{6}, z_{2}^{6}-\sqrt{2} z_{1}^{3} z_{2}^{3}+z_{1}^{6}, z_{1}^{2} z_{2}^{2}$, $z_{1} z_{2}$. By inspection, it is clear the considerably smaller subset of

$$
z_{1} z_{2}, \quad z_{1}^{3}+z_{2}^{3}, \quad z_{1}^{6}+z_{2}^{6}
$$

will generate the ring. 
Algorithm 3.2 has a significant simplification in the case of a diagonal unitary group $\Gamma$. In this case $\mathbb{C}[z]^{\Gamma}$ will have monomial generators. To see this, note that if

$$
\gamma=\left(\begin{array}{ccc}
\lambda_{1}^{\gamma} & & \\
& \ddots & \\
& & \lambda_{n}^{\gamma}
\end{array}\right),
$$

then

$$
1-\langle\gamma z, z\rangle=1-\lambda_{1}^{\gamma}\left|z_{1}\right|^{2}-\cdots-\lambda_{n}^{\gamma}\left|z_{n}\right|^{2}
$$

so (1.1) has the form

$$
\sum c_{\alpha \alpha}\left|z^{\alpha}\right|^{2}
$$

Algorithm 3.3. The case where $\Gamma$ is a unitary diagonal group.

1. Expand (1.1) into the form (3.6).

2. The set of monomials $z^{\alpha}$ which appear in (3.6) will be a set of generators for $\mathbb{C}[z]^{\Gamma}$.

As an example of Algorithm 2, consider the matrix group generated by

$$
\left(\begin{array}{ccc}
\omega & 0 & 0 \\
0 & \omega^{2} & 0 \\
0 & 0 & \omega^{4}
\end{array}\right),
$$

where $\omega$ is a sixth root of unity. This matrix group was examined by Grundmeier in this doctoral thesis [6]. With $x=\left|z_{1}\right|^{2}, y=\left|z_{2}\right|^{2}$, $z=\left|z_{3}\right|^{2}$, Grundmeier computed that

$$
\begin{aligned}
\Phi(z, \bar{z})= & 1-\prod_{\gamma \in \Gamma}(1-\langle\gamma z, z\rangle) \\
= & x^{6}+6 x^{4} y+9 x^{2} y^{2}+2 y^{3}-y^{6} \\
& +6 x^{2} z+6 y z-6 x^{2} y^{3} z-6 y^{4} z \\
& -3 x^{4} z^{2}-9 y^{2} z^{2}+2 z^{3}-2 y^{3} z^{3} \\
& +3 x^{2} z^{4}-6 y z^{4}-z^{6} .
\end{aligned}
$$


The 16 appearing terms will generate the invariant ring. By inspection, we note that the considerably smaller subset of the seven terms $x^{6}, y^{3}$, $z^{3}, x^{2} y^{2}, y z, x^{2} z, x^{4} y$ will also suffice.

4. Finite abelian groups. The finite abelian groups are notable because in this case the holomorphic decomposition diagonalization step can be eliminated. In this case our main theorem has an especially simple form.

Theorem 4.1. Let $\Gamma \leq G L(n, \mathbb{C})$ be a finite abelian group. Then there exists a $T \in G L(n, \mathbb{C})$ such that

$$
1-\prod_{\gamma \in \Gamma}(1-\langle T \gamma z, T z\rangle)=\sum c_{\alpha \alpha}\left|T(z)^{\alpha}\right|^{2},
$$

where the set of $\left\{T(z)^{\alpha}\right\}$ generates $\mathbb{C}[z]^{\Gamma}$.

In other words, by multiplying out

$$
1-\prod_{\gamma \in \Gamma}\left(1-\left\langle T \gamma T^{-1} z, z\right\rangle\right)=\sum c_{\alpha \alpha}\left|z^{\alpha}\right|^{2},
$$

the set $\left\{T(z)^{\alpha}\right\}$ gives a set of generators for the invariant ring.

Proof. Since $\Gamma$ is finite, each of its matrix elements will be of finite order and hence individually diagonalizable. Since $\Gamma$ is a commuting family of matrices, the elements of $\Gamma$ may be simultaneously diagonalized, see [8]. Thus, there exists a $T \in G L(n, \mathbb{C})$ such that

$$
\Gamma^{\prime}=T \Gamma T^{-1}
$$

is a group of diagonal matrices. Since the matrices are also of finite order, they must be unitary as well. Thus, by Theorem 3.1 and the remarks above about unitary diagonal representations,

$$
1-\prod_{\gamma \in \Gamma}\left(1-\left\langle T \gamma T^{-1} z, z\right\rangle\right)
$$

will be of the form of equation (3.6). The conclusion follows after making a change of variable $z \mapsto T z$. 
To see an example of how the invariants arise consider the abelian group

$$
V_{4}=\left\{ \pm\left(\begin{array}{ll}
1 & 0 \\
0 & 1
\end{array}\right), \pm\left(\begin{array}{ll}
0 & 1 \\
1 & 0
\end{array}\right)\right\} \subset G L(2, \mathbb{C}),
$$

a representation of the Klein four-group.

We begin by simultaneously diagonalizing the group by means of

$$
\left(\begin{array}{cc}
-1 & 0 \\
0 & 1
\end{array}\right)=T\left(\begin{array}{ll}
0 & 1 \\
1 & 0
\end{array}\right) T^{-1}
$$

where

$$
T=\frac{1}{\sqrt{2}}\left(\begin{array}{cc}
1 & 1 \\
1 & -1
\end{array}\right) .
$$

Thus, we obtain the equivalent unitary diagonal representation of the Klein four-group

$$
T V_{4} T^{-1}=\left\{ \pm\left(\begin{array}{ll}
1 & 0 \\
0 & 1
\end{array}\right), \pm\left(\begin{array}{cc}
-1 & 0 \\
0 & 1
\end{array}\right)\right\} .
$$

This gives, with $x=\left|z_{1}\right|^{2}$ and $y=\left|z_{2}\right|^{2}$,

$$
\begin{aligned}
\Phi & =1-[(1-x-y))(1+x+y)(1-x+y)(1+x-y)] \\
& =2 x^{2}+2 y^{2}+2 x^{2} y^{2}-x^{4}-y^{4},
\end{aligned}
$$

yielding the set

$$
\left\{x^{2}, y^{2}, x^{2} y^{2}, x^{4}, y^{4}\right\}
$$

that is,

$$
\left\{z_{1}^{2}, z_{2}^{2}, z_{1}^{2} z_{2}^{2}, z_{1}^{4}, z_{2}^{4}\right\}
$$

is a set of generators for the diagonal representation. By inspection, we see

$$
\left\{z_{1}^{2}, z_{2}^{2}\right\}
$$

will be a minimal generating set. Composing with $T$ gives as a generating set for the original representation (4.1)

$$
\left\{\frac{\left(z_{1}+z_{2}\right)^{2}}{2}, \frac{\left(z_{1}-z_{2}\right)^{2}}{2}\right\}
$$

or more simply $\left(z_{1}+z_{2}\right)^{2},\left(z_{1}-z_{2}\right)^{2}$. 
5. Closing remarks. By standard methods, it can be shown that the worst-case computational complexity of Algorithm 1 for computing a generating set for an invariant ring is somewhat worse than the classical Reynolds operator. This seems to mainly arise from the fact that (1.1) lives in a ring with double the number of variables as well as its fundamentally multiplicative nature. Since our main result, Theorem 1, is independent of Algorithm 1, it is conceivable that more efficient algorithms might exist. For example, consider our treatment of the dihedral group in (3.3) above. Note that we can write

$$
\Phi=\Phi_{2}+\Phi_{3}+\Phi_{4}+\Phi_{5}+\Phi_{6},
$$

where $\Phi_{2}=6 z_{1} z_{2} \overline{z_{1} z_{2}}, \Phi_{3}=z_{1}^{3}{\overline{z_{1}}}^{3}+z_{2}^{3}{\overline{z_{1}}}^{3}+z_{1}^{3}{\overline{z_{2}}}^{3}+z_{2}^{3}{\overline{z_{2}}}^{3}, \Phi_{4}=$ $-9 z_{1}^{2} z_{2}^{2}{\overline{z_{1}}}^{2} \overline{z^{2}}, \Phi_{5}=-3 z_{1}^{4} z_{2} \bar{z}_{1}^{4} \overline{z_{2}}-3 z_{1} z_{2}^{4} \bar{z}_{1}^{4} \overline{z_{2}}-3 z_{1}^{4} z_{2}{\overline{z_{1} z_{2}}}^{4}-3 z_{1} z_{2}^{4}{\overline{z_{1}}}_{z_{2}}{ }^{4}$, $\Phi_{6}=-z_{1}^{3} z_{2}^{3}{\overline{z_{1}}}^{6}-z_{1}^{6}{\overline{z_{1}}}^{3}{\overline{z_{2}}}^{3}-z_{2}^{6}{\overline{z_{1}}}^{3}{\overline{z_{2}}}^{3}-z_{1}^{3} z_{2}^{3} \bar{z}_{2}{ }^{6}$, that is, as a sum of bihomogeneous components. We could have likewise applied Algorithm 1 to each of the $\Phi_{i}$ and dealt with at worst, two $2 \times 2$ matrices and three $3 \times 3$ matrices (as opposed to one $9 \times 9$ matrix). In fact, more is true. Suppose we simplify each of the $\Phi_{i}$ by means of grouping and common factors as in $\Phi_{3}=z_{1}^{3}{\overline{z_{1}}}^{3}+z_{2}^{3}{\overline{z_{1}}}^{3}+z_{1}^{3}{\overline{z_{2}}}^{3}+z_{2}^{3}{\overline{z_{2}}}^{3}=$ $z_{1}^{3}\left(\bar{z}_{1}^{3}+\bar{z}_{2}^{3}\right)+z_{2}^{3}\left(\bar{z}_{1}^{3}+{\overline{z_{2}}}^{3}\right)=\left(z_{1}^{3}+z_{2}^{3}\right)\left({\overline{z_{1}}}^{3}+{\overline{z_{2}}}^{3}\right)=\left|z_{1}^{3}+z_{2}^{3}\right|^{2}$. Likewise, $\Phi_{2}=6\left|z_{1} z_{2}\right|^{2}, \Phi_{4}=-9\left|z_{1} z_{2}\right|^{2}$ and $\Phi_{5}=-3\left|z_{1}^{4} z_{2}+z_{1} z_{2}^{4}\right|^{2} . \Phi_{6}$ does not simplify in quite as straightforward a way but can be handled using the technique of analytic polarization as follows:

$$
\begin{aligned}
\Phi_{6} & =-z_{1}^{3} z_{2}^{3}{\overline{z_{1}}}^{6}-z_{1}^{6}{\overline{z_{1}}}^{3}{\overline{z_{2}}}^{3}-z_{2}^{6}{\overline{z_{1}}}^{3}{\overline{z_{2}}}^{3}-z_{1}^{3}{z_{2}^{3}}^{3}{\overline{z_{2}}}^{6} \\
& =-\left[z_{1}^{3} z_{2}^{3}\left(\bar{z}_{1}^{6}+{\overline{z_{2}}}^{6}\right)+z_{1}^{6}{\overline{z_{1}}}^{3}{\overline{z_{2}}}^{3}+z_{2}^{6}{\overline{z_{1}}}^{3}{\overline{z_{2}}}^{3}\right] \\
& =-\left(z_{1}^{6}+z_{2}^{6}\right)\left(\bar{z}_{1}^{3}{\overline{z_{2}}}^{3}\right) \\
& =-2 \operatorname{Re}\left\{\left(z_{1}^{6}+z_{2}^{6}\right) z_{1}^{3} z_{2}^{3}\right\} \\
& =\frac{\left|z_{1}^{3} z_{2}^{3}-z_{1}^{6}-z_{2}^{6}\right|^{2}}{2}-\frac{\left|z_{1}^{3} z_{2}^{3}+z_{1}^{6}+z_{2}^{6}\right|^{2}}{2} .
\end{aligned}
$$

The last equality was obtained by the well-known polarization identity

$$
\operatorname{Re}(z \bar{w})=\frac{|z+w|^{2}-|z-w|^{2}}{4}
$$

for complex numbers $z$ and $w$. See $[\mathbf{3}, 4]$ for more on polarization as a means of computing holomorphic decompositions. Collectively, these give a holomorphic decomposition of $\Phi$ and yield generating invariants which are essentially the same as (3.5). Analytic polarization, however, 
need not give the correct invariants unless the component polynomials are linearly independent, as is true in the above case. Its applicability to the invariant generation problem is thus not completely understood.

We should make some remarks on the diagonalization step in Algorithm 3.2. In elementary linear algebra, diagonalization is carried out by first computing the eigenvalues of the matrix, using these eigenvalues to compute the eigenspaces and then converting to an orthonormal basis using Gram-Schmidt (or some other orthogonalization algorithm) if necessary. In practice, the characteristic equation may be of large degree which may impede our ability to find the eigenvalues. As an alternative approach, we could employ algorithms from numerical linear algebra to compute the eigenvalues numerically and obtain a set of algebra generators with numerical approximations to the coefficients. Another alternative would be carry out the diagonalization symbolically, expressing the generators by allowing the eigenvalues $\lambda_{1}, \lambda_{2}, \ldots$ to appear symbolically (i.e., as parameters) in our final output.

Acknowledgments. The authors would like to thank the National Science Foundation for support of the University of Wisconsin-Stout REU held during the summer of 2012 .

\section{REFERENCES}

1. M. Salah Baouendi, Peter Ebenfelt and Linda Preiss Rothschild, Real submanifolds in complex space and their mappings, Princeton University Press, Princeton, 1999.

2. Albert Boggess, CR Manifolds And The Tangential Cauchy-Riemann complex, CRC Press, Boca Raton, 1991.

3. John P. D'Angelo, Several complex variables and the geometry of real hypersurfaces, CRC Press, Boca Raton, 1992.

4. Inequalities in complex analysis, The Carus Math. Mono. 28, Mathematical Association of America, Washington, D.C., 2002.

5. Steven Deckelman, An invariant-theoretic property of certain CR mappings, Complex Var. Elliptic Eq. 59 (2014), 120-132.

6. Dusty Grundmeier, Group-invariant $\mathrm{CR}$ mappings, $\mathrm{PhD}$ thesis, University of Illinois, Urbana, Illinois, 2011.

7. Signature pairs of group-invariant Hermitian polynomials, Inter. J. Math. 22 (2011), 311-343.

8. Roger A. Horn and Charles R. Johnson, Matrix analysis, Cambridge University Press, Cambridge, 1990. 
9. Mara Neusel, Invariant theory, American Mathematical Society, Providence R.I., 2007.

10. Joseph Wolf, Spaces of constant curvature, 4th ed., Publish or Perish, Berkeley, California, 1977.

Department of Mathematics, Statistics and Computer Science, University of Wisconsin-Stout, Menomonie, WI 54751

Email address: deckelmans@uwstout.edu

Department of Mathematics, Statistics and Computer Science, University of Wisconsin-Stout, Menomonie, WI 54751

Email address: graetzjl@gmail.com

Department of Mathematics, Statistics and Computer Science, University of Wisconsin-Stout, Menomonie, WI 54751

Email address: tyler.russell1113@gmail.com 